\title{
Decarboxylative Protonation of Allyl Ester Catalyzed by Pt and Ru
}

\author{
Saravanan Gowrisankar, Eun Sun Kim, and Jae Nyoung Kim \\ Department of Chemistrv and Institute of Basic Science. Chonmam National Chiversitw, Gwangiu 500-757, Korea \\ E-mail: kiminachomam.ac.kr \\ Received Wovember 7, 2008. Accepted November 28. 2008
}

Key Words: Decarboxylative protonation. Platinum, Grubbs catalyst

After the Tsuji's brilliant contribution in the area of Pd-cataly $<$ ed decarboxylative protonation and allylation. 'many research groups used the reactions in a variety of sy nthesis. ${ }^{23}$ For decarboxylative protonation the combination of $\mathrm{HCOOH} / \mathrm{Et}_{3} \mathrm{~N}$ was ussed most frequently as the hydrogen source. '. Recently Meldrum's acid ${ }^{3 a}$ and water ${ }^{3}$ have been used as the hydrogen donor. Recently we reported an efficient synthesis of 1.5-dicarbonyl compounds from Baylis-Hillman adducts by using Pd-mediated decarbosylative protonation strategy. ${ }^{3}$ In the reaction we used aqueous $\mathrm{CH}_{3} \mathrm{CN}$ as the reaction medium to provide the hydrogen atom. ${ }^{3}$

Although many transition metals can form the corresponding $\pi$-allylmetal complexs. ${ }^{+}$there was no report involving the use of other metals except palladium in the decarboxylative protonation of allyl ester. ${ }^{1-3}$ Thus, we decided to examine the feasibility of decarboxy lative protonation with $1 \mathrm{a}$ as a model substrate. which was used in our previous paper. ${ }^{3}$

As summarized in Table l we examined some metal cataly'sts which can be easily available commercially. These included four platinum catalysts and two Grubbs catalysts $\left(1^{3 t}\right.$ and $2^{\text {tid }}$ generation). Platinum catalysts were all effective in the reactions as in entries 2-4 and 9. However. relatively longer reaction time was required than the Pd-catalyzed reaction (entry 1) . The use of TPP (triphenylphosphine) was cnicial in these reactions. "When we replace TPP into dppe [1,2-bis(diphenylphosphino)ethane]. dppp [1.3-bis(diphenylphosphino) propane,$n-B u_{3} \mathrm{P}$ and ( $o$-tolyl) ${ }_{3} \mathrm{P}$. the yield of product was decreased (entries 5-8). The reason is not clear at this moment. It is interesting to note that two Gnibbs catalysts were also effective. ${ }^{6}$ However, somew hat elevated temperature was required for the effective reaction in these cases (entries 10 and 11). As in entries 12 and $13, \mathrm{Ni}(\mathrm{OAc})_{2}$ and $\mathrm{Cu}(\mathrm{OAc})$. were less effective even with $10 \mathrm{~mol} \%$ of catalyst.

Based on the experimental observations we chose two conditions (entries 4 and 10 ) as the comparable conditions to the Pd-mediated reaction (entry 1$)^{3}$ in view of the yield of product and easy availability of the catalyst. Under the selected conditions decarboxylative protonations were examined with 1b and 1c, and the results are summarized in Scheme 1. From the reaction of $1 \mathrm{~b}$ we isolated decarboxylative protonation product 2b as the major (75-76\%) in all cases. Acid derivative 3b was isolated in small amounts under Pd-and Pt-catalyzed conditions. Decarboxylative protonation product $2 \mathrm{c}$ was obtained again as the major ( $74-85 \%$ ) from the reaction of $1 \mathrm{c}$. Decarbosylative allylation product $3 \mathrm{c}$ was also obtained in small amounts $(5-10 \%)$. and compound $4 \mathrm{c}$ was isolated in $11 \%$ when we used
Pd as the catalyst. Although the results showed slight differences depending on the catalyst. major products were decarboxylative protonation products in all cases and the yields of them were similar.

From the comparative studies of 1a-c with $\mathrm{Pd}(\mathrm{OAc})_{2}$. $\mathrm{K}_{2} \mathrm{PtCl}_{4}$. and Grubbs catalyst. decarboxylative protonation reaction could be carried out with either one of the catalysts. Further studies on the application of this condition to different substrates and the synthetic applicability of Grubbs catalyst are currently undervay.

Table 1. Decarboxylative protonation of 1 a to 2 a catalyzed by Pt or $\mathrm{Ru} .{ }^{a}$
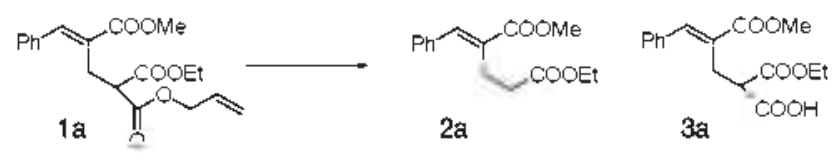

\begin{tabular}{|c|c|c|c|c|}
\hline \multirow{2}{*}{ Entry } & \multirow{2}{*}{$\begin{array}{c}\text { Catalyst } \\
(5 \%)\end{array}$} & \multirow{2}{*}{$\begin{array}{l}\text { Ligand } \\
(10 \%)\end{array}$} & \multirow{2}{*}{$\begin{array}{c}\text { Time } \\
\text { (h) }\end{array}$} & \multirow{2}{*}{$\begin{array}{c}\text { Yield (\%) } \\
\mathbf{1} \mathbf{a} / \mathbf{2 a} / \mathbf{3 a}\end{array}$} \\
\hline & & & & \\
\hline $1^{R e f ?}$ & $\mathrm{Pd}(\mathrm{OAc})_{2}$ & $\mathrm{TPP}$ & 2 & $0 / 90 / 0$ \\
\hline 2 & $\mathrm{PtO}_{2}$ & TPP & 13 & $0 / 88 / 0$ \\
\hline 3 & $\mathrm{Pt}\left(\mathrm{PPh}_{3}\right)_{4}$ & TPP & 15 & $0 / 90 / 0$ \\
\hline 4 & $\mathrm{~K}_{2} \mathrm{PtCl}_{+}$ & $\mathrm{TPP}$ & 15 & $0 / 90 / 0$ \\
\hline 5 & $\mathrm{~K}_{2} \mathrm{PtCl}_{+}$ & dppe & 15 & $38 / 53 / 0$ \\
\hline 6 & $\mathrm{~K}_{2} \mathrm{PtCl}_{4}$ & dppp & 12 & $58 / 31 / 0$ \\
\hline 7 & $\mathrm{~K}_{2} \mathrm{PtCl}_{4}$ & $n-\mathrm{Bu}_{3} \mathrm{P}$ & 12 & $67 / 24 / 0$ \\
\hline 8 & $\mathrm{~K}_{2} \mathrm{PtCl}_{+}$ & $\mathrm{P}(0-\mathrm{tol} y 1)_{3}$ & 15 & $4 / 79 / 10$ \\
\hline 9 & $\mathrm{PtCl}_{2}$ & $\mathrm{TPP}$ & 15 & $6 / 82 / 10$ \\
\hline $10^{b}$ & Grubbs $1^{\text {st }}$ & TPP & 15 & $0 / 80 / 0$ \\
\hline $11^{b}$ & Grubbs $2^{\text {ild }}$ & TPP & 15 & $0 / 77 / 0$ \\
\hline 12 & $\mathrm{Ni}\left(\mathrm{OAc}_{2}\right)^{c}$ & TPP & 15 & $36 / 53 / 0$ \\
\hline 13 & $\mathrm{Cu}(\mathrm{OAc})_{2}{ }^{c}$ & $\mathrm{TPP}$ & 15 & $34 / 61 / 0$ \\
\hline
\end{tabular}

${ }^{a}$ All reactions were carried out in the presence of $\mathrm{Et}_{3} \mathrm{~N}$ ( 1.2 equiv) in $\mathrm{CH}_{3} \mathrm{CN} / \mathrm{H}_{2} \mathrm{O}(9: 1)$ at $70^{\circ} \mathrm{C}$. "Reaction was run under refluxing conditions. Grubbs $1^{\text {sl }}$ : benzylidene-bis(tricyloherylphosphine) dichlororuthenium. Grubbs $2^{\text {thl. }}:$ 1.3-bis-(2.4.6-trimethylphenyl)-2-imidazoliinylidene) dichlore(phenylmethylene)(tricyclohexylphosphine) ruthenium. "Catalyst 10\%. 
<smiles>CCOC(=O)C(CCc1ccccc1)C(=O)O</smiles>

$1 \mathrm{~b}$<smiles>CC(=O)C(Cc1ccccc1)C(=O)O</smiles><smiles>CCOC(=O)CCCc1ccccc1</smiles>

2b<smiles>CCOC(=O)C(CCc1ccccc1)C(=O)O</smiles>

3b

$\mathrm{Pd}(\mathrm{OAc})_{2} / \mathrm{TPP} / 70^{\circ} \mathrm{C}: 2 \mathrm{~b}(75 \%), 3 \mathrm{~b}(7 \%)$ $\mathrm{K}_{2} \mathrm{PtCl}_{4} / \mathrm{TPP} / 70^{\circ} \mathrm{C}: 2 \mathrm{~b}(76 \%), 3 \mathrm{~b}(8 \%)$ Grubbs $1^{\text {st }} /$ TPP/reflux: 2b $(75 \%)$, 3b (0\%)

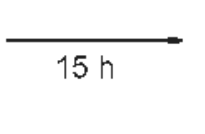

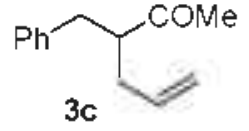

2c<smiles>CC(=O)/C=C/c1ccccc1</smiles>

$4 \mathrm{c}$

$\mathrm{Pd}(\mathrm{OAC})_{2} / \mathrm{TPP} / 70^{\circ} \mathrm{C}: 2 \mathrm{c}(74 \%), 3 \mathrm{C}(10 \%), 4 \mathrm{c}(11 \%)$ $\mathrm{K}_{2} \mathrm{PtCl}_{4} / \mathrm{TPP} 70^{\circ} \mathrm{C}: 2 \mathrm{C}(82 \%), 3 \mathrm{c}(5 \%), 4 \mathrm{c}$ (not formed) Grubbs 15 st/TPP/reflux: $2 c(85 \%), 3 c(7 \%), 4 c$ (not formed)

Scheme 1

\section{References and Notes}

1. For Tsuji contribution on Pd-assisted decarbosylative protonation and allylation, see: (a) Tsuji, J; Nisar, M.; Shimizu, I. $J$. Org. Chem. 1985, 50, 3416-3417. (b) Mandai, T.; Imaii, M.: Takada, H.; Kawata, M.: Nokami, I.; Tsuij, J. J. Org. Chem 1989. 54, 5395-5397. (c) Tsuji, J. Pure Appl. Chem 1986, 58, 869-878.

2. For the other contributions on $\mathrm{Pd}$-assisted decarboxylative protonation, see: (a) Marinescu, S. C.: Nishimata, T: Molu, J. T.; Stoltz, B. M. Org. Lett. 2008, 10, 1039-1042. (b) Ragoussis, V.; Giannikopoulos, A. Tetrahedron Lett. 2006, 47, 683-687.

3. Our recent contribution, Gowrisankar, S.: Kim, K. H.; Kim, S. H.; Kim, J. N. Tetrahedron Lett. 2008, 19, 6241-6244.

4. For some examples on the formation of $\pi$-ally lmetal complex and their synthetic applications, see: (a) Blacker, A. T.; Clarke, M. L.; Loft, M. S.: Williams, J. M. J. Chem. Conmm 1999, $913-914$. (b) Blacker, A. I.; Clarke, M. L.; Loft, M. S.; Mahon, M. F.: Humphries, M. E.: Williams, I. M. J. Chent. Ent J. 2000, 6 , 353-360. (c) Kadota, T: Komori, S: Fukumoto, Y.; Murai, S. $J$. Org. Chem 1999, 64, 7523-7527, (d) Kadota, T.: Katsuragi, H.: Fukumoto, Y.: Murai, S. Organometallics 2000, 19,979-983. (e) Utsunomiya, M.; Miyamoto, Y.; Ipposhi, T.: Ohshima, T.; Mashima, K. Org. Lett. 2007, 9, 3371-3374. (f) Kitamura, M; Tanaka, S.; Yoshimura, M. J. Ory. Chem. 2002, 67, 4975-4977. (g) Burger, E. C.: Tunge, T. A Chem. Commm 2005, 2835-2837. (h) Constant, S.: Tortoioli, S.: Muller, I.; Lacour, J . Angew. Chem. Iht. Ed. 2007, t6, 2082-2085. (i) Burger, E. C.; Tunge, J. A. Org. Lett. 2004, 6, 2603-2605. (i) Corey, E. T.: William, T. J. Org. Chem. 1973, 38, 3223-3224. (k) Tunge, J. A.: Burger, E. C. Enr: J. Org. Chem. 2005, 1715-1726 and further references cited therein.

5. Without TPP the reactions with $\mathrm{PtO}_{2}$, Grubbs catalysts, Ni(OAc)s and $\mathrm{Cu}(\mathrm{OAC}$ ) were very sluggish. However, appreciable amounts of product was fonmed with $\mathrm{Pd}(\mathrm{OAc})_{2}, \mathrm{Pt}\left(\mathrm{PPl}_{2}\right)_{4}, \mathrm{~K}_{2} \mathrm{PtCl}_{4}$ and $\mathrm{PtCl}_{2}$ without TPP.

6. For Ru-catalyzed deallylation, see: (a) Alcaide, B : Almendros, P.: Alonso, I. M. Chem. Eur. J. 2006, I2, 2874-2879. (b) Alcaide,
B.; Almendros, P.: Alonso, I. M. Tetrahedron Lett. 2003, 4t. 8693-8695. (c) Tanaka, S.: Saburi, H.; Kitamura, M. Adv. Synth. Cotal. 2006, 348, 375-378. (d) Tanaka, S.; Hirakawa, T.; Oishi, K.; Hayahawa, Y.; Kitamura, M. Tetrohedron Lett. 2007, 48 , 7320-7322. (e) Saburi, H.; Tanaka, S.; Kitamura, M. Angetw. Chem. Int. Ed 2005, H4, 1730-1732. (f) Tanaka, S.; Saburi, H.; Murase, T.; Ishibashi, Y; Kitamura, M. J. Organometal Chem. 2007, 692, 295-298

7. Typical procedure for the synthesis of $2 \mathrm{a}$ (entry 4 ): To a stirred solution of $1 \mathrm{a}(173 \mathrm{mg}, 0.5 \mathrm{mmol}), \mathrm{K}_{2} \mathrm{PtCl}_{4}$ ( $1 \mathrm{ll} \mathrm{mg}, 5 \mathrm{~mol} \%$ ), $\mathrm{PPh}_{3}(14 \mathrm{mg}, 10 \mathrm{~mol} \%)$ in $\mathrm{CH}_{3} \mathrm{CN}_{2} \mathrm{H}_{2} \mathrm{O}(3 \mathrm{~mL}, 9: 1)$ was added $\mathrm{Et}_{3} \mathrm{~N}(6 \mathrm{lmg}, 0.6 \mathrm{mmol})$ and the reaction mixture was heated to 70 "C for $15 \mathrm{~h}$. After usual aqueous workup and column chromatographic purification process (hexanes/ether, 95:5) compound 2a was isolated as colorless oil, $118 \mathrm{mg}(90 \%)$. $^{3}$ Other compounds were synthesized similarly and the representative spectroscopic datá of $1 \mathrm{~b} .1 \mathrm{c}$. and $3 \mathrm{c}$ are as follows.

Compound 1b: colorless oil; IR ( film) $2939,1752,1734 \mathrm{~cm}^{\cdot 1}:{ }^{1} \mathrm{H}$ $\mathrm{NMR}\left(\mathrm{CDCl} l_{3}, 300 \mathrm{MHz}\right) \delta 1.27(\mathrm{t}, J=7.2 \mathrm{~Hz}, 3 \mathrm{H}), 2.20-2.28(\mathrm{~m}$. $2 \mathrm{H}), 2.64-2.69(\mathrm{~m}, 2 \mathrm{H}), 3.38(\mathrm{t}, J=7.5 \mathrm{~Hz}, 1 \mathrm{H}), 4.2 \mathrm{l}(\mathrm{q}, J=7.2$ $\mathrm{Hz}, 2 \mathrm{H}), 4.62-4.65(\mathrm{~m}, 2 \mathrm{H}), 5.22-5.36(\mathrm{~m}, 2 \mathrm{H}), 5.84-5.97$ (m, lH), 7.17-7.34 (m, 5H) ${ }^{13} \mathrm{CNMR}\left(\mathrm{CDCl}_{3}, 75 \mathrm{MHz}\right) \delta 14.07,30.34$, $33.29,51.20,61.43,65.85,118.58,126.20,128.45,128.52,131.59$, $140.57,169,00,169.16$.

Compound 1c: colorless oil; IR (tilm) $3030,1743,1716 \mathrm{~cm}^{13} ;{ }^{1} \mathrm{H}$ NMR $\left(\mathrm{CDCl}_{3}, 300 \mathrm{MHz}\right) \delta 2.18(\mathrm{~s}, 3 \mathrm{H}), 3.17(\mathrm{~d}, J=7.8 \mathrm{~Hz}, 2 \mathrm{H})$. $3.86(\mathrm{t}, J=7.8 \mathrm{~Hz}, \mathrm{lH}), 4.564 .59(\mathrm{~m}, 2 \mathrm{H}), 5.18-5.27(\mathrm{~m}, 2 \mathrm{H})$, $5.75-5.88(\mathrm{~m}, 1 \mathrm{H}), 7.14-7.29(\mathrm{~m}, 5 \mathrm{H}),{ }^{13} \mathrm{C} \mathrm{NMR}\left(\mathrm{CDCl}_{3}, 75 \mathrm{MHz}\right)$ $829.58,33.89,61.10,65.86,118.78,126.60,128.48,128.68,131.29$, $137.93,168.64,202.13$.

Compound 3c: colorless oil; IR (tilm) 2924, $1712 \mathrm{~cm}^{-1}$ : ${ }^{1} \mathrm{HNMR}$ $\left.(\mathrm{CL}) \mathrm{Cl}_{3}, 300 \mathrm{MHz}\right) \delta 1.99(\mathrm{~s}, 3 \mathrm{H}), 2.17-2.26(\mathrm{~m}, \mathrm{lH}), 2.31-2.4 \mathrm{l}$ $(\mathrm{m}, 1 \mathrm{H}), 2.66-2.75(\mathrm{~m}, 1 \mathrm{H}), 2.85-2.95(\mathrm{~m}, 2 \mathrm{H}), 5.02-5.09(\mathrm{~m}, 2 \mathrm{H})$ $5.65-5.79(\mathrm{~m}, 1 \mathrm{H}), 7.13-7.30(\mathrm{~m}, 5 \mathrm{H}) \cdot{ }^{13} \mathrm{CNMR}\left(\mathrm{CDCl}_{3}, 75 \mathrm{MHz}\right)$ $830.48,35.65,37.32,54.14,117.20,126.30,128.45,128.84,135.09$, $139.37,211.45$ 